\title{
Managing the paediatric patient with amelogenesis imperfecta
}

\author{
S. McDonald, ${ }^{1}$ N. Arkutu, ${ }^{2}$ K. Malik, ${ }^{3}$ K. Gadhia ${ }^{4}$ and S. McKaig ${ }^{5}$
}

IN BRIEF
- This paper discusses the problems
experienced by children with
amelogenesis imperfecta.
- Discusses the challenges of providing
dental care for these children.
- Provides clinical guidance for managing
amelogenesis imperfecta in the primary,
mixed and permanent dentitions.

A diagnosis of amelogenesis imperfecta can present challenges for patient and clinician alike. This can be further complicated in a child due to cooperation and the ever-changing dentition. This paper looks at the ideals for management at various stages of the developing dentition but also considers the compromises which may have to be made depending on the child's ability to cope. The importance of early diagnosis, prevention and the benefits of a multi disciplinary approach are also discussed.

\section{INTRODUCTION}

The challenges faced in managing a patient with amelogenesis imperfecta (AI) are numerous. This is further magnified when considering the paediatric patient. AI affects both the primary and secondary dentitions. ${ }^{1}$ The clinical issues are sensitivity (particularly to cold), rapid wear and poor aesthetics, all of which can vary depending on the type of AI. ${ }^{1}$ Treatment is aimed at addressing these problems, relieving symptoms, maintaining face height and managing aesthetics. Other anomalies associated

\section{AMELOGENESIS IMPERFECTA \\ 1. Amelogenesis imperfecta: an introduction \\ 2. Managing the paediatric patient with amelogenesis imperfecta \\ 3. Amelogenesis imperfecta: the orthodontic perspective \\ 4. The interdisciplinary management of patients with amelogenesis imperfecta - restorative dentistry}

'Specialist Registrar in Paediatric Dentistry, Birming ham Dental Hospital, St Chad's Queensway, Birmingham; ${ }^{2}$ Consultant Orthodontist, University Hospital of North Staffordshire NHS Trust, Orthodontic Department, Hartshill Road, Stoke-on-Trent; ${ }^{3}$ Consultant in Restorative Dentistry, Birmingham Dental Hospital, St Chad's Queensway, Birmingham; ${ }^{4 *}$ Specialist Registrar in Restorative Dentistry, Eastman Dental Hospital, 256 Gray's Inn Road, London; ${ }^{5}$ Consultant in Paediatric Dentistry, Birmingham Dental Hospital, St Chad's

Queensway, Birmingham

*Correspondence to: Kushal Gadhia

Email: kushalgadhia@nhs.net; Tel: 02034561154

\section{Refereed Paper}

Accepted 13 January 2012

DOI: 10.1038/sj.bdj.2012.366

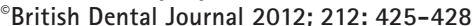

with AI include disturbances in eruption, anterior open bite, pulpal calcifications, pathological root or crown resorption and taurodontism, ${ }^{1-3}$ all of which can present in the paediatric patient. Added to the difficulties of managing $\mathrm{AI}$ are the issues of a child's dental inexperience, self-perception, dental anxiety and parental expectation, which further complicate its management.

The paper will aim to discuss the management of the paediatric patient presenting with AI.

\section{MANAGEMENT}

As with all children, initial experience of the dental profession plays a part in their cooperation for the future. Children with AI often require extensive dental treatment throughout their lives, therefore it is essential to ensure the initial experiences are positive. However, this can be hard to achieve when a child may be experiencing sensitivity when brushing their teeth and eating/drinking cold things. Seeing a child as early as possible will enable the clinician to build up a rapport with the child and parents/carers.

Consideration needs to be given as to when and how appointments are arranged to try and support the family, as multiple visits will impact on schooling and be a challenge for working parents.

\section{PREVENTION}

Prevention is the most important tool in any clinician's armamentarium. ${ }^{4}$ It is particularly important when teeth are known

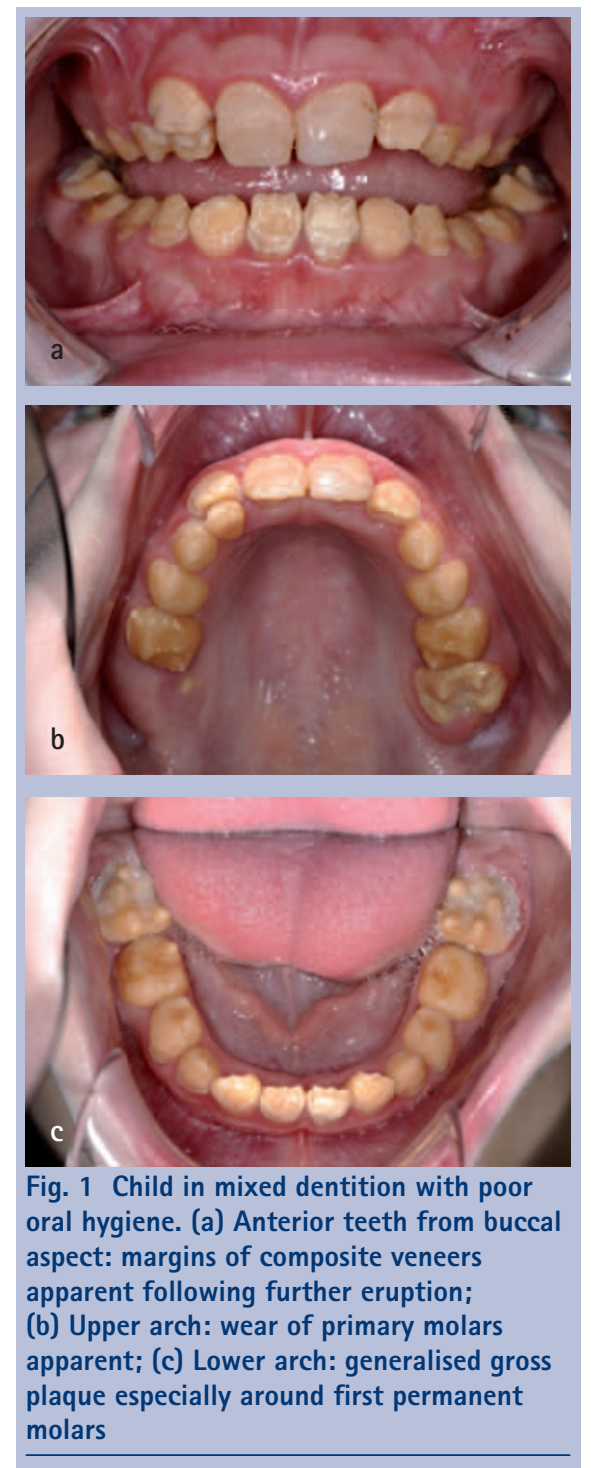

to be vulnerable, as is the case in AI. Oral hygiene can be particularly difficult for this group of patients due to sensitivity 
when brushing. Advising the use of warm water for tooth brushing will go some way to relieving symptoms when rinsing. Regular use of fluoride mouthwashes can also help to reduce sensitivity and prevent caries in children old enough to be trusted not to swallow it. ${ }^{5}$ However, the discomfort associated with brushing can not always be overcome and cases where there are significant plaque and calculus deposits are not unusual (Fig. 1). Good periodontal health is important for long term success of treatment. ${ }^{6}$

Dietary analysis and advice regarding erosion and sugars are fundamental to reducing further problems in teeth affected by AI. Anecdotally, children will often avoid ice cream and fridge-cold products but this still leaves a big array of other cariogenic or acidic products for them to choose. Counselling needs to be given to the child and family to explain why the child is at increased risk and encourage the whole family to support the affected child.

\section{TREATMENT}

Like any child, a child with AI may need restorations and scaling; however, sensitivity can be a challenge to providing this treatment. Local anaesthesia (LA) is an essential tool in helping these children receive treatment. Even a simple, fine scale may require LA over a few visits. The hygienist or therapist can form an essential part of the team by providing this care at regular intervals. ${ }^{6}$ They can also assist with motivation, continuing preventative advice and support. ${ }^{7}$ One must also consider sensitivity of other teeth when providing a restoration, as although the tooth being treated is anaesthetised, it is not uncommon for the child to complain of sensitivity on the opposite side of the mouth. Providing treatment using non-latex dam can reduce the sensitivity experienced during treatment.

With regard to managing AI in a child, the aim should be to manage any symptoms or complaints the child may have. Radiographs are a valuable tool in assessing caries but can also provide information regarding thickness and quality of enamel. Delayed eruption, taurodontism and idiopathic resorption (Fig. 2) may also be identified. Where there is no evidence of wear, minimal intervention and monitoring is the preferred treatment plan. This
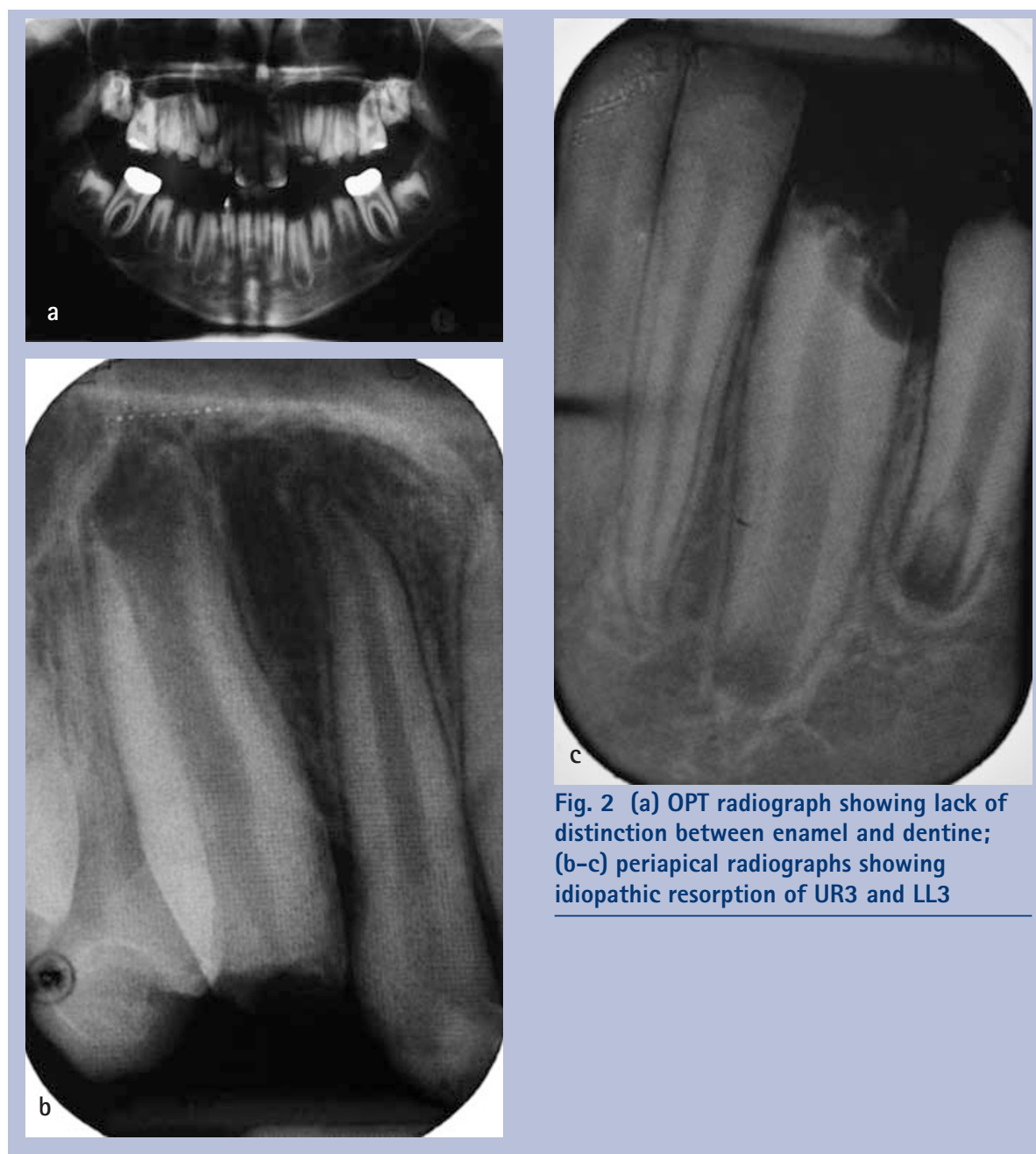

Fig. 2 (a) OPT radiograph showing lack of distinction between enamel and dentine; (b-c) periapical radiographs showing idiopathic resorption of UR3 and LL3

will make treatment decisions for the adult patient a lot easier. However, when a child is experiencing sensitivity, occlusal wear or complains about their aesthetics then intervention becomes necessary.

\section{PRIMARY DENTITION}

In a child requiring management of their primary dentition the following should be considered:

- That the treatment provided reflects the degree of symptoms or wear experienced

- Glass ionomer or composite direct veneers of anterior teeth to address aesthetics

- Stainless steel crowns (SSC) or glass ionomer restorations on the occlusal surfaces of primary molars, where it is not possible to place SSC. Orthodontic separators between molars will assist placement of SSC. ${ }^{8}$

No tooth preparation is required to undertake this treatment, assuming the dentition is caries free. In an anxious or young child compromise may be necessary, in which case:

- Second deciduous molars are considered to be higher priority to maintain the occlusion (Fig. 3)

- Placing aesthetic veneers on anterior teeth may be used as acclimatisation and help to motivate the child and family. However, there is always a risk that the child may not return as their primary complaint may be addressing the aesthetics.

\section{MIXED DENTITION}

The permanent teeth will also be affected by $\mathrm{AI}$ and need to be assessed and possibly protected as soon as is practicable. Management considerations at this stage are:

- Gold onlays/crowns (depending on the clinical indication or sensitivity of the teeth ${ }^{1}$ ) or SSC for first permanent molars when they have fully erupted ${ }^{9}$ (Fig. 4). SSC require less cooperation from the child and also have the advantage of being completed in one visit. However, gold restorations will 

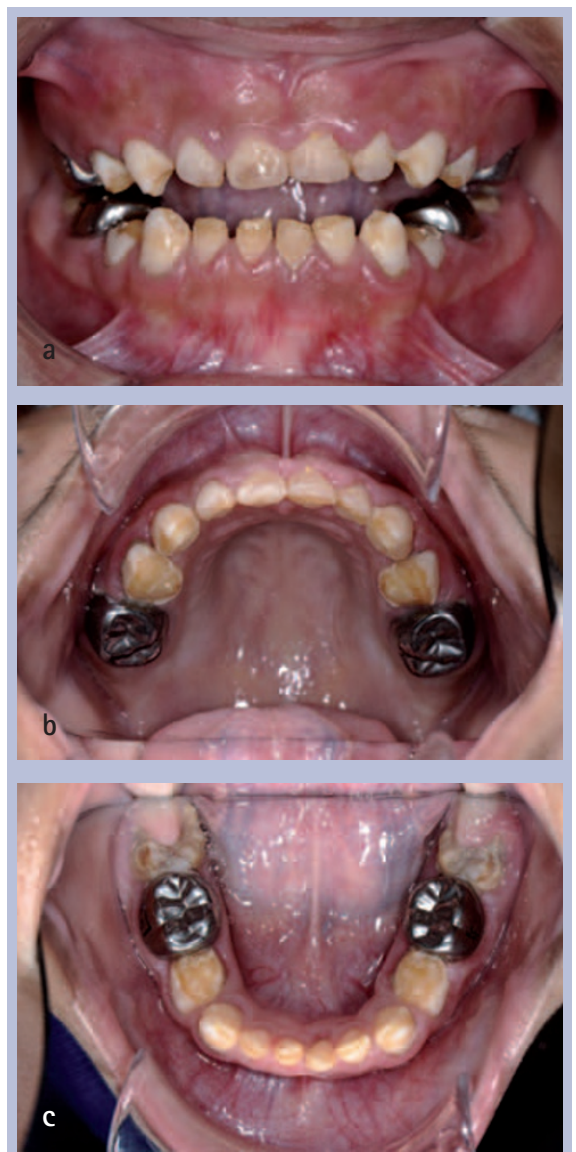

Fig. 3a-c Early mixed dentition showing stainless steel crowns in situ. Enamel break down is already apparent on the partially erupted lower first molars

be more definitive and the margins can be kept supragingivally to aid hygiene and gingival health ${ }^{9}$

- Teeth can take a protracted time to erupt and thus damage can occur while waiting. In this situation, one may need to consider placing glass ionomer occlusally until full eruption or in protracted cases, consider excising the residual operculum to expose the whole crown and then proceed with restorations

- For permanent incisors direct or indirect composite veneers will improve aesthetics, reduce sensitivity and reduce incisal wear. However, a child can still be relatively young when permanent incisors erupt and so it may still require an individual to become more mature and self aware before self motivation for treatment increases. Direct composites have the advantage that they can be started as soon as there is sufficient tooth erupted and added to as further eruption occurs. However, the
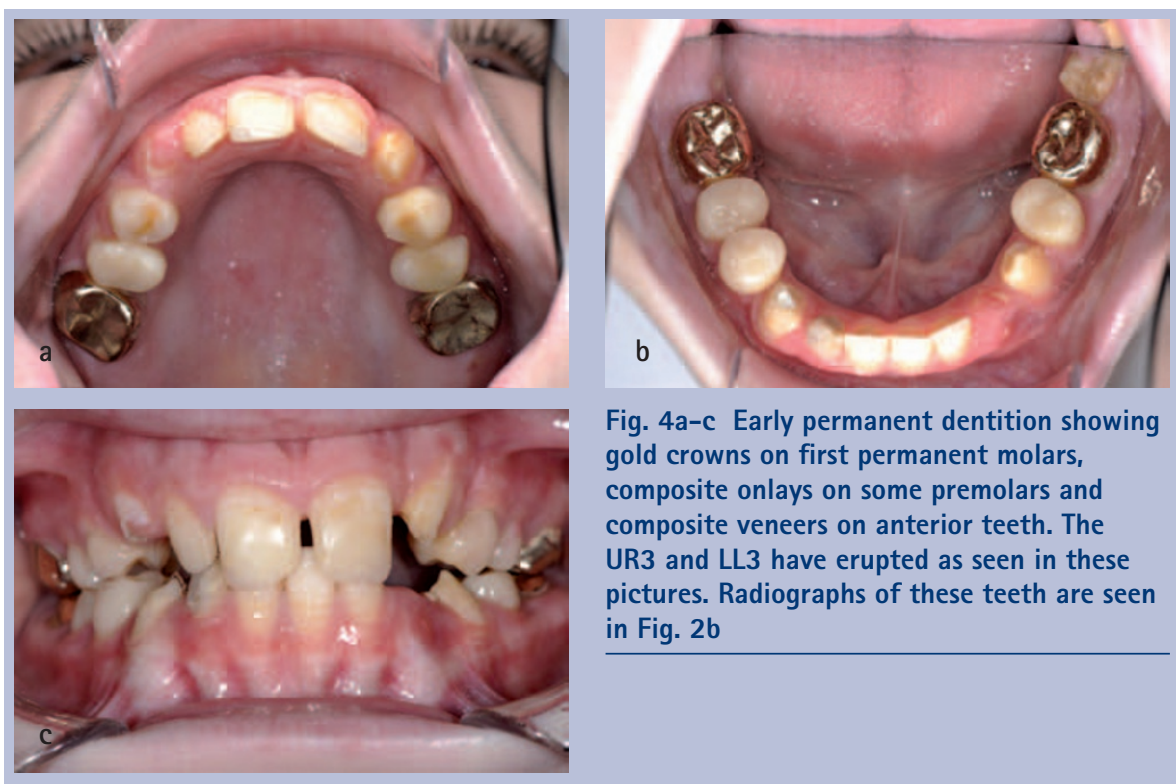

Fig. 4a-c Early permanent dentition showing gold crowns on first permanent molars, composite onlays on some premolars and composite veneers on anterior teeth. The UR3 and LL3 have erupted as seen in these pictures. Radiographs of these teeth are seen in Fig. $2 b$

child and parent should be warned that with continuing eruption and gingival maturation, the margins of any restoration will become visible and additional treatment will be required, at intervals, to maintain good aesthetics (Fig. 1a).

\section{PERMANENT DENTITION}

With regard to premolars and permanent canines, it should be remembered that minimal intervention is the ideal treatment plan.

If premolars are not in occlusion and they are not particularly sensitive then no intervention may be required. The aesthetics of premolars are often not a concern.

When wear and sensitivity are an issue, full coronal coverage can be provided with indirect or direct composite onlays.

Canines are more likely to be an aesthetic concern in addition to sensitivity and wear. As with permanent incisors, composite can solve these problems.

As with the primary dentition, permanent tooth preparation is not usually needed because one of the aims of treatment is to preserve as much tooth structure as possible. It is also important to remember immature and young teeth have very large pulp chambers. When the teeth are more developed, tooth preparation and porcelain can be considered for defınitive restorations.

It is important to monitor the permanent dentition as it starts to mature. Delayed eruption and resorption of teeth need to be identified and consideration given to management (Figs 2b and 4). Options for resorbing teeth include extraction of the affected teeth or attempting to orthodontically extrude them before too much resorption occurs. An orthodontic option can also be considered for failure of eruption but these issues will be further discussed in the third paper of this series.

Definitive management of the dentition can not occur until full dental and gingival maturity, that is, adulthood. It is the responsibility of the dentist managing the child's dental care to help maintain the dentition until that time. However, early consideration of the long term plan for the individual can ensure that one is working towards the long term objective, thus the opinions of the orthodontist and restorative dentist are valuable. Joint speciality clinics can be very useful for this cohort of children to ensure a multi disciplinary approach.

\section{BEHAVIOUR MANAGEMENT}

Children with AI can be dentally anxious like any other child, however, these children have the added pressures of AI. The use of behavioural management techniques and sedation will be of great value to the clinician and patient alike. ${ }^{10}$ In some children it may be the only way to achieve any treatment. Even so, some children cannot manage dental treatment of the simplest nature while awake. In children with such severe anxiety it may be necessary to consider general anaesthesia (GA). Although this may seem drastic in a child that has no infection or dental caries, 
failure to protect the dentition at this early stage may well result in a dentition too poor to restore in adulthood. However, one must always bear in mind that using GA is not a risk-free procedure.

\section{DISCUSSION}

There is usually a family history of AI so parents may well know what their child will have to go through to maintain their dentition. ${ }^{8}$ When discussing the management of AI, a clinician must obviously discuss the ideal management of the child but may have to modify the treatment plan dependent upon the child's cooperation. Naturally, a parent will want what is best for their child and in some cases, may put pressure on the child and clinician to achieve the ideal. This can be a very difficult situation for all involved and the clinician may have to become an advocate for the child. The study from Coffield et al. demonstrated that AI has a detrimental effect on perceived wellbeing in adulthood, ${ }^{6}$ which may also be the case in childhood. However, it is imperative to look towards the long term goal of a well-adjusted dental patient who has a pain free, aesthetic dentition in adulthood rather than creating a dental phobic.

Children with AI can have high dental needs and may present many dental challenges. Understanding, patience, behavioural management skills and sedation are valuable tools for helping a child to maintain their dentition. Ensuring a child is pain free is essential. However, improvement of aesthetics is probably greeted with as much enthusiasm by the child and family. Prevention of excessive wear should also be a priority to ensure that there is sufficient dentition to restore in adulthood. A multi disciplinary team involving general dental practitioners, paediatric dentists, orthodontists, restorative colleagues, therapists and hygienists can be beneficial in managing this cohort of patients.
1. Ayers K M, Drummond B K, Harding W J, Salis S G, Liston P N. Amelogenesis imperfecta - multidisciplinary management from eruption to adulthood. Review and case report. NZDent J 2004; 100: 101-104.

2. Poulson $\mathrm{S}, \mathrm{Gjør}$ p $\mathrm{H}_{1}$ Haubek D et al. Amelogenesis imperfecta - a systematic literature review of associated dental and oro-facial abnormalities and their impact on patients. Acta Odontol Scand 2008; 66: 193-199.

3. Crawford P J, Aldred M, Bloch-Zupan A. Amelogenesis imperfecta. Orphanet J Rare Dis 2007; 2: 17.

4. Department of Health. Delivering better oral health: an evidence based toolkit for prevention - second edition. Department of Health and the British Association for the Study of Community Dentistry, 2009.

5. Marinho V C. Cochrane reviews of randomized trials of fluoride therapies for preventing dental caries Eur Arch Paediatr Dent 2009; 10: 183-191.

6. Coffield K D, Phillips C, Brady M, Roberts M W, Strausse R P, Wright J T. The psychosocial impact of developmental dental defects in people with hereditary amelogenesis imperfecta. J Am Dent Assoc 2005; 136: 620-630.

7. Luzzi V Bossù E, Cavellè E, Ottolenghi L, Polimeni A. Case report: clinical management of hypoplastic amelogenesis imperfecta. Eur J Paediatr Dent 2003; 4: $149-154$.

8. Kwok-Tung L, King N M. The restorative management of amelogenesis imperfecta in the mixed dentition. J Clin Pediatr Dent 2006; 31: 130-135.

9. Zagdwon A M, Fayle S A, Pollard M A. A prospective clinical trial comparing preformed metal crowns and cast restorations for defective first permanent molars. Eur J Paediatr Dent 2003; 4: 138-142.

10. Hosey M T. Managing anxious children: the use of conscious sedation in paediatric dentistry. Int $J$ Paediatr Dent 2002; 12: 359-372. 